\title{
SIMPLY INVARIANT SUBSPACES AND GENERALIZED ANALYTIC FUNCTIONS ${ }^{1}$
}

\author{
T. P. SRINIVASAN
}

Let $(X, m)$ be a probability measure space and $A$ a subalgebra of $L^{\infty}(d m)$ on which $m$ is "multiplicative," meaning,

$$
\int f g d m=\int f d m \int g d m
$$

for all $f, g \in A$. Let $A_{0}$ be the set of functions in $A$ with $\int f d m=0$. Denote by $H^{p}(d m)$ the closure $[A]_{p}$ of $A$ in $L^{p}(d m), p=1,2$ and by $H^{\infty}(\mathrm{dm})$ the weak* closure $[A]_{*}$ of $A$ in $L^{\infty}(\mathrm{dm})$. We shall drop the parenthesis $(d m)$, in the future, while referring to $L^{p}(d m) H^{p}(d m)$, etc. The functions in $H^{p}$ we call generalized analytic functions.

Say that a closed subspace $\mathfrak{M}$ of $L^{p}$ is simply invariant if $\left[A_{0}, \mathfrak{M}\right]_{p}$ $\subset \mathfrak{M}$ and the inclusion is strict. For logmodular algebras $A$ it was shown in [5] that the simply invariant subspaces of $L^{p}$ have the form $q H^{p}$ where $q \in L^{p}$ and $|q|=1$ a.e. We shall refer to this result as the " $L^{p_{-}}$ invariant subspace theorem." The proof in [5] also shows that the $\operatorname{logmodularity}$ of $A$ is inessential for the truth of this theorem, that the $L^{1}$-theorem follows from the $L^{2}$-theorem and the following two conditions are sufficient for the truth of the $L^{2}$-theorem: tion.

$\mathrm{H}_{1} . A+\bar{A}$ is dense in $L^{2}$ where the bar denotes complex conjuga-

$\mathrm{H}_{2}$. If $f \in L^{1}, f \geqq 0$ and if $\int f g d m=0$ for all $g \in A_{0}$ then $f=c$ a.e. for some constant $c$.

It turns out that these two conditions are necessary as well for the validity of the $L^{2}$-theorem. One of our purposes in this paper is to prove this (Corollaries 1.1, 2.4). The key to our proof is a factorization theorem (Theorem 2) used (but not explicitly stated) by us in [5] to derive the $L^{1}$-invariant subspace theorem from the $L^{2}$-theorem. We derive on the way, from this factorization theorem, several consequences on generalized analytic functions which were proved by Hoffman [3] in the special case of logmodular algebras; Hoffman's machinery was different and more elaborate. Our proof of the $L^{1}$ invariant subspace theorem in [5] had some gaps. We rederive this theorem here (Corollary 2.5) for completeness. The $L^{1}$-theorem in

Received by the editors June 22, 1963 and, in revised form, May 21, 1964.

1 This work was done while I held a visiting appointment at the University of California, Berkeley. I thank Professors Henry Helson and Ju-kwei Wang for the useful discussions I had with them. 
its turn leads to an $L^{\infty}$-invariant subspace theorem (Theorem 3 ) which as an immediate corollary yields a maximality theorem for $H^{\infty}$ (Corollary 3.1). In case $X$ is the circle $|z|=1, m$ the Lebesgue measure and $A$ the algebra of analytic trigonometric polynomials, this maximality theorem was proved by Hoffman $[4$, p. 193] using a theorèm of Gleason-Whitney. In [1] Bochner extended the theorems established by Helson Lowdenslager in [2] to a general class of algebras. The algebras considered by Bochner satisfy our hypotheses $\mathrm{H}_{1}$ and $\mathrm{H}_{2}$ above [6], [7] so that our results are valid in Bochner's set up. Thus this paper captures some of the results considered by Bochner and by Hoffman and offers some new results in a set up more general than and by a machinery different from theirs. The only hypothesis in our set up is the validity of the $L^{2}$-invariant subspace theorem or its equivalent assumptions $\mathrm{H}_{1}$ and $\mathrm{H}_{2}$ stated earlier.

Since writing this paper we have been able to show in collaboration with Professor Ju-kwei Wang that essentially all the results of Bochner [1] and of Hoffman [3] are valid in their full strength in our set up and that no weaker assumption than ours can yield any of their major theorems. These and other cognate results will be published separately.

We assume that $A$ is a subalgebra of $L^{\infty}$ on which $m$ is multiplicative and that the $L^{2}$-invariant subspace theorem is true for $A$. We shall use the symbol $q$ with or without subscripts to denote a measurable function with absolute value 1 a.e. Let

$H_{0}^{p}=\left\{f \mid f \in H^{p}, \int f d m=0\right\}, H_{0}^{\infty}=\left\{f: f \in H^{\infty}, \int f d m=0\right\}$.

It is easy to see that $H_{0}^{p}=\left[A_{0}\right]_{p}$, and $H_{0}^{\infty}=\left[A_{0}\right]_{*}$. We have

TheOREM 1.

$$
L^{2}=H^{2} \oplus \bar{H}_{0}^{2} .
$$

Proof. Let $\mathfrak{M}=L^{2} \ominus H_{0}^{2}$. Then $\mathfrak{M}$ is a simply invariant subspace of $L^{2}$, so $\mathfrak{M}=q H^{2}$ for some $q$. Since $\mathfrak{M}$ contains constants, $\bar{q} \in H^{2}$. Let $\bar{c}=\int \bar{q} d m$. Then $\bar{q}-\bar{c} \in H_{0}^{2}$, so $q-c \in \bar{H}_{0}^{2}$. But $q, c$ and hence $q-c \in \mathfrak{M}$ and so $q-c \perp \bar{H}_{0}^{2}$. Hence $q-c=0$ and $\mathfrak{M}=H^{2}$.

Corollary 1.1. $A+\bar{A}$ is dense in $L^{2}$ (and hence in $L^{p}$ for $1 \leqq p \leqq 2$ ).

COROLlary 1.2 .

$$
H^{1} \cap L^{2}=H^{2} .
$$

For, if $f \in H^{1} \cap L^{2}$ then $f \perp \bar{A}_{0}$ and hence also $f \perp \bar{H}_{0}^{2}$. So $f \in H^{2}$. This 
shows $H^{1} \cap L^{2} \subset H^{2}$. The reverse inclusion is trivial.

We shall say that $h \in H^{p}$ is outer if $[h A]_{p}=H^{p}, p=1,2$. The following factorization theorem is very useful for applications.

THEOREM 2. If $f \in L^{1}$ and $f \notin\left[f A_{0}\right]_{1}$, then $f=F h$ where $h \in H^{2}$ is outer with $|h|^{2}=|f|$ and $F \in[f A]_{1} \cap L^{2}$.

Proof. Factor $f$ as $f_{1} f_{2}$ where $f_{1}=(\operatorname{sgn} . f)|f|^{1 / 2}$ and $f_{2}=|f|^{1 / 2}$. Then $f_{i} \in L^{2}, i=1,2$. Now $f_{2} \notin\left[f_{2} A_{0}\right]_{2}$, otherwise $f=f_{1} f_{2} \in f_{1}\left[f_{2} A_{0}\right]_{2} \subset\left[f A_{0}\right]_{1}$, contrary to our assumption. Hence $\left[f_{2} A\right]_{2}$ is a simply invariant subspace of $L^{2}$. Let $\left[f_{2} A\right]_{2}=q_{2} H^{2}$. Then $f_{2}=q_{2} h$ for some $h \in H^{2}$, outer, and $|h|^{2}=\left|f_{2}\right|^{2}=|f|$. Let $F=f_{1} q_{2}$. Trivially $F \in L^{2}$. Also

$$
F=f_{1} q_{2} \in f_{1} q_{2} H^{2}=f_{1}\left[f_{2} A\right]_{2} \subset[f A]_{1} .
$$

Of course $f=F h$.

REMARKs. (i) The proof shows that we need only assume that $|f|^{1 / 2} \notin\left[|f|^{1 / 2} A_{0}\right]_{2}$ in place of $f \notin\left[f A_{0}\right]_{1}$ for the validity of the theorem. Actually the two conditions are equivalent both being equal to $\int \log |f| d m>-\infty$ but we shall not prove this here.

(ii) An outer function with given modulus is easily seen to be unique (up to multiplication by a constant of absolute value 1 ). It follows that the $h$ above is uniquely determined by $f$. We may call $h$ the outer part of $f$.

CoRollary 2.1. If $f \in L^{1}, f \in\left[f A_{0}\right]_{1}$ then $f=q h^{2}$ for some $q$ where $h$ is the outer part of $f$.

For, then $[F A]_{2}$ is simply invariant subspace of $L^{2}$ and hence $[F A]_{2}=q H^{2}$ for some $q$. So $F=q h^{\prime}$ where $h^{\prime} \in H^{2}$ is outer. Since $\left|h^{\prime}\right|=|f|^{1 / 2}=|h|$ and $h^{\prime}, h$ are both outer we may assume that $h^{\prime}=h$. [3].

For logmodular algebras this corollary is essentially Theorem 5.9 in

Corollary 2.2. If $f \in H^{1}, f \in\left[f A_{0}\right]_{1}$ then $f=h_{1} h_{2}$ where $h_{i} \in H^{2}$ for $i=1,2 .^{2}$

For, then $F \in[A f]_{1} \cap L^{2} \subset H^{1} \cap L^{2}=H^{2}$.

Corollary 2.3. If $f \in L^{1}$ and $\int f g d m=0$ for all $g \in A_{0}$ then $f \in H^{1}$ (and trivially conversely).

For, by considering $f+c$ for some constant $c$ if necessary, we can

2 I owe this remark to Professor Calvin Moore. 
assume that $\int f d m \neq 0$. Then $f \notin\left[f A_{0}\right]_{1}$ and so by the main theorem, $f=F h$. Since $F \in[A f]_{1}$ it follows that $\int F g d m=0$ for all $g \in A_{0}$. Also $F \in L^{2}$. So $F \in H^{2}$ by Theorem 1 . Hence $f \in H^{1}$.

For logmodular algebras this is Theorem 6.1 in [3]; the proof in [3] is involved.

Corollary 2.4. If $f \in L^{1}, f \geqq 0$ and $\int f g d m=0$ for all $g \in A_{0}$ then $f=c$ a.e. for some constant $c$.

For, we can assume as in Corollary 2.3 that $\int f d m \neq 0$ so that $f \notin\left[f A_{0}\right]_{1}$. Using the notation in the proof of Theorem 2, we have then $f=F h$ where $F=f^{1 / 2} q_{2}$ and $f^{1 / 2}=q_{2} h$. As in Corollary 2.3, $F \in H^{2}$. It follows that both $f^{1 / 2} q_{2}$ and $f^{1 / 2} \bar{q}_{2} \in H^{2}$. This implies by Theorem 1 that $f^{1 / 2} q_{2}=c$ for some constant $c$. Then $f^{1 / 2}=|c|$, so $f$ is a constant a.e.

CoROLlARY 2.5. The $L^{1}$-invariant subspace theorem: If $\mathfrak{M}$ is a simply invariant subspace of $L^{1}$ then $\mathfrak{M}=q H^{1}$ for some $q$.

For, let $\mathfrak{N}=\mathfrak{M} \cap L^{2}$ and $f \in \mathfrak{M} \backslash\left[A_{0} \mathfrak{M}\right]_{1}$, where $\backslash$ denotes the set theoretic difference. Then $f=F h$ by Theorem 2, where $h \in H^{2}$ and $F \in \mathfrak{M} \cap L^{2}=\mathfrak{N}$. Also $f \in\left[A_{0} \mathfrak{N}\right]_{2}$, for otherwise,

$$
f=F h \in\left[A_{0} \mathfrak{N}\right]_{2} h \subset\left[A_{0} \mathfrak{N}\right]_{1} \subset\left[A_{0} \mathfrak{M}\right]_{1},
$$

contrary to our assumption. It follows that $\mathfrak{N}$ is simply invariant. Let $\mathfrak{N}=q H^{2}$. We shall show that $\mathfrak{M}=q H^{1}$. From the above we can conclude that $\mathfrak{M} \backslash\left[A_{0} \mathfrak{M}\right]_{1} \subset q H^{1}$. Also the algebraic sum

$$
\left[A_{0} \mathfrak{M}\right]_{1}+\left(\mathfrak{M} \backslash\left[A_{0} \mathfrak{M}\right]_{1}\right) \subset\left(\mathfrak{M} \backslash\left[A_{0} \mathfrak{M}\right]_{1}\right) .
$$

Hence $\left[A_{0} \mathfrak{M}\right]_{1} \subset q H^{1}$. Hence $\mathfrak{M} \subset q H^{1}$. The reverse inclusion is trivial.

REMARK. It can be shown that the $L^{1}$-invariant subspace theorem is in fact equivalent to the $L^{2}$-theorem. We shall not prove this here.

We introduced $H^{\infty}$ as the weak* closure of $A$. We note the following

LEMMA.

$$
H^{\infty}=H^{1} \cap L^{\infty}=H^{2} \cap L^{\infty} .
$$

Proof. Only the first equality is nontrivial. Clearly $H^{1} \cap L^{\infty} \supset A$. It is immediate from Corollary 2.3 that $H^{1} \cap L^{\infty}$ is weak ${ }^{*}$ closed. It follows that $H^{1} \cap L^{\infty} \supset H^{\infty}$. If the inclusion were strict, by the HahnBanach Theorem there would exist a linear functional $I$ on $L^{\infty}$ which is zero on $H^{\infty}$ but not on $H^{1} \cap L^{\infty}$. Let $I$ be realized by the $L^{1}$ function $f$. Then, for every $g \in A$

$$
I(g)=\int g f d m=0 .
$$


By Corollary 2.3, $f \in H_{0}^{1}$. This implies that $\int g f d m=0$ for all $g \in H^{1} \cap L^{\infty}$, contradicting our assumption on $I$. Hence we conclude that $H^{1} \cap L^{\infty}$ $=H^{\infty}$.

For any subset $\mathfrak{M}$ of $L^{\infty}$, let

$$
\mathfrak{M}^{\perp}=\left\{f \in L^{1}: \int f \ddot{g} d m=0 \text { for all } g \in \mathfrak{M}\right\} .
$$

For any two subsets $\mathfrak{M}$, $\mathfrak{N}$ we shall write $\mathfrak{M}<\mathfrak{N}$ to signify that $\mathfrak{M} \subset \mathfrak{N}$ and the inclusion is strict. We shall say that a subspace $\mathfrak{M}$ of $L^{\infty}$ is simply invariant if it is weak* closed and $\left[A_{0} \mathfrak{M}\right]_{*}<\mathfrak{M}$. We have

TheOREM 3 ( $L^{\infty}$-INVARIANT SUBSPACE THEOREM). Every simply invariant subspace $\mathfrak{M}$ of $L^{\infty}$ is of the form $q H^{\infty}$ (and trivially conversely).

Proof. Let $\mathfrak{M}_{1}=\left[A_{0} \mathfrak{M}\right]_{*}$. Then $\overline{\mathfrak{M}}_{1}^{\perp}$ (where the bar denotes complex conjugation) is clearly a closed subspace of $L^{1}$ and is invariant for multiplication by functions in $A$. Also $A_{0} \overline{\mathfrak{M}}_{1}^{\perp} \subset \overline{\mathfrak{M}}^{\perp}<\overline{\mathfrak{M}}_{1}^{\perp}$. The last inequality results from our hypothesis $\mathfrak{M}_{1}<\mathfrak{M}$, by an application of the Hahn-Banach theorem. It follows that $\mathfrak{M}_{1}^{\perp}$ is a simply invariant subspace of $L^{1}$ and by Corollary 2.5, is of the form $\bar{q} H^{1}$. Then $\mathfrak{M}_{1}$ $=q H_{0}^{\infty}$. In particular, $\bar{q} A_{0} \mathfrak{M} \subset H_{0}^{\infty}$ so that for every $f \in \bar{q} \mathfrak{M}, \int f g d m=0$ for all $g \in A_{0}$; it follows by Corollary 2.3 and the lemma above that $\bar{q} \mathfrak{M} \subset H^{1} \cap L^{\infty}=H^{\infty}$. Thus

$$
q H_{0}^{\infty}=\mathfrak{M l}_{1}<\mathfrak{M} \subset q H^{\infty} .
$$

The extreme spaces differ by dimension 1 ; hence $\mathfrak{M}=q H^{\infty}$.

Corollary 3.1..$^{3}$ Any weak* closed subalgebra of $L^{\infty}$ which contains $H^{\infty}$ and is simply invariant, coincides with $H^{\infty}$.

For, let $B$ be such an algebra. By Theorem 3, $B=q H^{\infty}$ for some $q$. Since $B$ is an algebra containing $q, q^{2} \in B=q H^{\infty}$; so $q \in H^{\infty}$. Since $B$ contains constants, $\bar{q} \in H^{\infty}$. It follows that $q$ is a constant a.e. Hence $B=H^{\infty}$.

In the special case when $X$ is the circle $|z|=1, m$, the (normalized) Lebesgue measure and $A$, the algebra of analytic trigonometric polynomials, if $B$ is any weak* closed subalgebra of $L^{\infty}$ which contains $H^{\infty}$, then either $B$ is simply invariant and the above corollary applies or $z B=B$, so $B$ contains $\bar{z}$ and its powers together with $z$ and its powers; and hence $B=L^{\infty}$. Thus in this case we have

Corollary 3.2. $H^{\infty}$ (of the circle) is a maximal weak* closed subalgebra of $L^{\infty}$.

This arose out of a discussion with Professor Ju-kwei Wang. 
We do not know whether this strengthened version of Corollary 3.1 is true in the general case.

Next we show that $H^{\infty}$ is a logmodular algebra on the maximal ideal space $\Omega$ of $L^{\infty}$. We recall that a subalgebra $B$ of the algebra $C(X)$ of all (complex) continuous functions on a compact Hausdorff space $X$ is logmodular if

(i) $B$ is uniformly closed, contains constants and separates points;

(ii) the set of functions $\log |f|$, where $f, 1 / f \in B$, is uniformly dense in $C_{R}(X)$, the algebra of real continuous functions on $X$.

We have

Theorem 4. The algebra $H^{\infty}$ with the m-essential supnorm is a logmodular algebra on the maximal ideal space $\Omega$ of $L^{\infty}$.

Proof. Only the condition (ii) above needs to be verified. Let $u$ be any real valued function in $L^{\infty}$. Clearly $e^{u} \notin e^{u} H_{0}^{2}$, so $e^{u} H^{2}$ is a simply invariant subspace of $L^{2}$. Let $e^{u} H^{2}=q H^{2}$. Then $e^{u}=q h$ for some $h \in H^{2} \cap L^{\infty}=H^{\infty}$. Since $q=e^{u} h^{\prime}$ for some $h^{\prime} \in H^{2}$ we have $h h^{\prime}=1$; so $h$ is invertible in $H^{\infty}$. Also $u=\log |h|$. This shows that $H^{\infty}$ is logmodular. [3].

For logmodular algebras $A$ this is the Corollary to Theorem 6.4 in

\section{REFERENCES}

1. S. Bochner, Generalized conjugate and analytic functions without expansions, Proc. Nat. Acad. Sci. U.S.A. 45 (1959), 855-857.

2. H. Helson and D. Lowdenslager, Prediction theory and Fourier series in several variables, Acta Math. 99 (1958), 165-202.

3. K. Hoffman, Analytic functions and logmodular Banach algebras, Acta Math. 108 (1962), 271-317.

4. - Banach spaces of analytic functions, Prentice-Hall, Englewood Cliffs, N. J., 1962.

5. T. P. Srinivasan, Simply invariant subspaces, Bull. Amer. Math. Soc. 69 (1965), 706-709.

6. - Doubly invariant subspaces, Pacific J. Math. 14 (1964), 701-707.

7. T. P. Srinivasan and Morisuke Hasumi, Doubly invariant subspaces. II, Pacific J. Math. 14 (1964), 525-535.

UNiversity of California, BERKEley aNd Panjab University, Chandigarh, India 